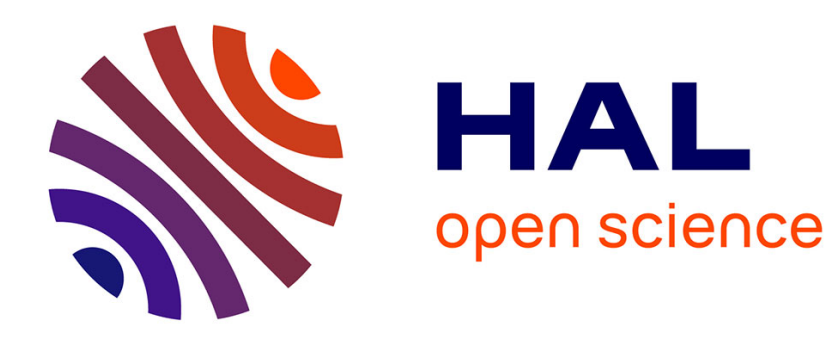

\title{
Contact interactions at HERA, LEP and Tevatron C. Goy
}

\section{To cite this version:}

C. Goy. Contact interactions at HERA, LEP and Tevatron. International Europhysics Conference on High Energy Physics EPS 2003, Jul 2003, Aachen, Germany. pp.s776 - s778, 10.1140/epjcd/s200303-822-8 . in2p3-00014086

\section{HAL Id: in2p3-00014086 https://hal.in2p3.fr/in2p3-00014086}

Submitted on 30 Oct 2003

HAL is a multi-disciplinary open access archive for the deposit and dissemination of scientific research documents, whether they are published or not. The documents may come from teaching and research institutions in France or abroad, or from public or private research centers.
L'archive ouverte pluridisciplinaire HAL, est destinée au dépôt et à la diffusion de documents scientifiques de niveau recherche, publiés ou non, émanant des établissements d'enseignement et de recherche français ou étrangers, des laboratoires publics ou privés. 
LAPP-EXP 2003-14

October 2003

Contact interactions at HERA LEP and TEVATRON

\author{
C. Goy
}

LAPP-IN2P3-CNRS

9 chemin de Bellevue - BP. 110

F-74941 Annecy-le-Vieux Cedex

Presented at the International Europhysics Conference on

High Energy Physics EPS, Aachen, Germany, July 17-23, 2003 


\section{CONTACT INTERACTIONS AT HERA, LEP AND TEVATRON}

Corinne Goy

LAPP Chemin de Bellevue, F 74941 Annecy-Le-Vieux

Received: date / Revised version: date

Abstract. Current experimental limits on 4-fermions Contact Interactions at Hera, LEP and Tevatron are reviewed.

\section{Introduction}

Four fermions Contact Interactions (CI) parametrise interactions beyond the Standard Model by an effective energy scale $\Lambda$. The new Lagrangian is of the form [1]:

$$
\mathrm{L}^{\mathrm{CI}}=\frac{4 \pi}{\Lambda_{\text {ef }}^{2}} \sum \eta_{\mathrm{ij}}\left(\mathrm{e}^{\mathrm{i}} \gamma^{\mu} \mathrm{e}_{\mathrm{i}}\right)\left(\mathrm{f}^{\mathrm{j}} \gamma_{\mu} \mathrm{f}_{\mathrm{j}}\right)
$$

where parameters $\eta_{i j}$ 's specify the relative contributions of the helicity states of the initial (e) and final (f) fermions involved in the new interaction, defining several models. The most commonly referenced models are listed in the table 1 . The new current can have a constructive or de-

Table 1. Definition of the most common CI models.

\begin{tabular}{lllllllll}
\hline & LL & RR & LR & RL & VV & AA & $\begin{array}{l}\text { V0 } \\
(\mathrm{X} 3)\end{array}$ & $\begin{array}{l}\text { A0 } \\
(\mathrm{X} 4)\end{array}$ \\
\hline$\eta_{L L}$ & 1 & 0 & 0 & 0 & 1 & 1 & 1 & 0 \\
$\eta_{R R}$ & 0 & 1 & 0 & 0 & 1 & 1 & 1 & 0 \\
$\eta_{L R}$ & 0 & 0 & 1 & 0 & 1 & -1 & 0 & 1 \\
$\eta_{R L}$ & 0 & 0 & 0 & 1 & 1 & -1 & 0 & 1 \\
\hline
\end{tabular}

structive interference with the Standard Model, and limits are labelled $\Lambda^{+}$and $\Lambda^{-}$.

Experiments operating on the colliders Hera, LEP and Tevatron are sensitive to couplings between different initial and final state fermions.

\section{Hera}

Hera is sensitive to e-q interactions and due to the parton distribution inside the proton, essentially to e-u and e-d couplings. Limits are derived from the differential crosssection of the neutral current $d \sigma^{N C} / d Q^{2}$ and exploit data taken in the configurations $e^{-} p$ at $\sqrt{s}=319 \mathrm{GeV}$ and $e^{+} p$ at $\sqrt{s} 301 \mathrm{GeV}$ and $319 \mathrm{GeV}$, cumulating a total luminosity close to $120 \mathrm{pb}^{-1}$ per experiment. H1 has finalised its analysis [2] of the Neutral Current and from the absence of deviation with respect to the predictions, limits ranging between 1.6 to $5.5 \mathrm{TeV}$ on $\Lambda^{-}$and between 2.8 to $5.3 \mathrm{TeV}$ on $\Lambda^{+}$are obtained. Figure 1 details the limits for several models.

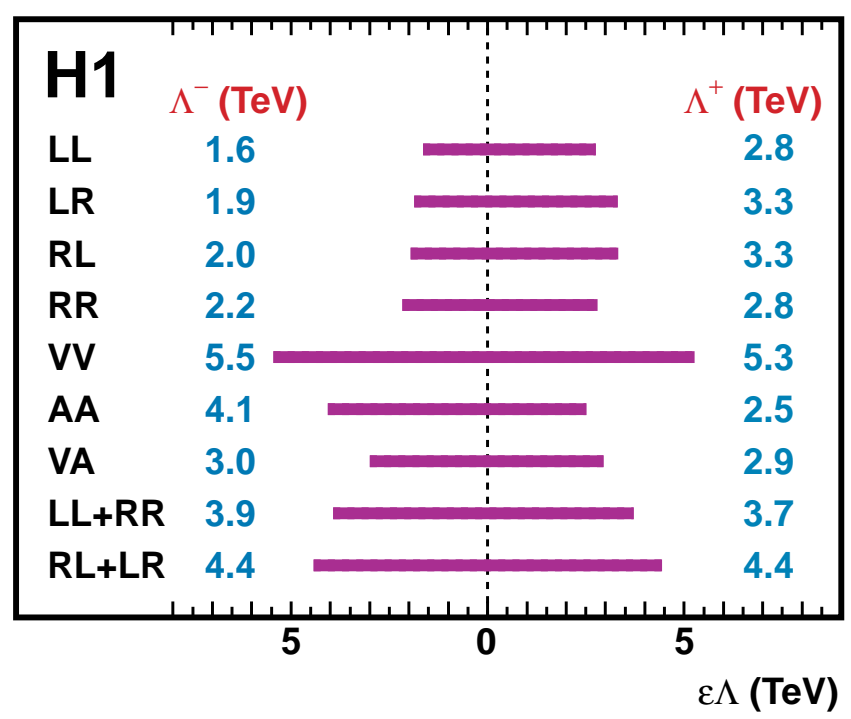

Fig. 1. Final limits from $\mathrm{H} 1$ on several models of Contact Interactions [2].

\section{Tevatron}

CDF and D0 have a unique sensitivity to 4 quarks and $q-\mu$ couplings, involving essentially valence quarks. With a luminosity of $110 \mathrm{pb}^{-1}$ per experiment taken at Run I at $\sqrt{s}=1.8 \mathrm{TeV}$, limits of the order of 2 to $5 \mathrm{TeV}$ [3],[4] could be set on $\Lambda$, using the di-jet or di-muons mass distributions. 


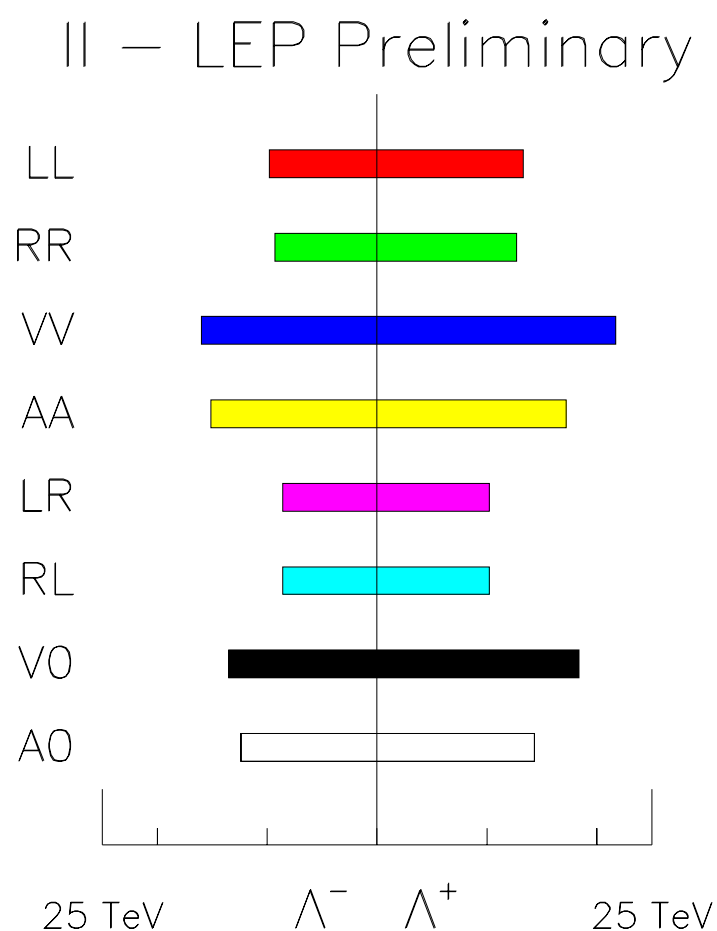

Fig. 2. Limits on the scale of CI in $e^{+} e^{-} \rightarrow \mu^{+} \mu^{-}+\tau^{+} \tau^{-}$ using LEP combined measurements from 130 to $209 \mathrm{GeV}$

\section{LEP}

The LEP experiments are sensitive to 4 leptons interaction, heavy quarks final state and neutrinos final state. Contact Interactions indirect effects could be seen in total and differential hadronic and leptonic cross-sections, leptonic asymmetries or heavy flavours observables $R_{b}, R_{c}$, $A_{F B}^{b}$ and $A_{F B}^{c}$. The $4 \mathrm{LEP}$ experiments cumulated a total luminosity close to $2.8 \mathrm{fb}^{-1}$ taken at centre-of-mass energies ranging between 130 to $209 \mathrm{GeV}$.

The preliminary LEP-combined measurements [5] of the electroweak observables did not show any deviation from the Standard Model predictions. Therefore limits on $\Lambda^{+}$and $\Lambda^{-}$were derived. Some results are shown in figure 2 for the combined $\mu^{+} \mu^{-}$and $\tau^{+} \tau^{-}$final state and in figure 3 for the bb final states.

LEP is also sensitive to CI with neutrino final state through the single photon topology. Delphi has performed such an interpretation [6] using the parametrisation $\varepsilon \times G_{F}$ $\left(\approx 4 \pi / 2 \sqrt{2} \Lambda^{2}[7]\right)$ The exclusion contours obtained in 3 different hypothesis for the final state $\left(\nu_{e} \overline{\nu_{e}}, \nu_{\tau} \overline{\nu_{\tau}}, \nu_{\alpha} \nu_{\beta}\right)$ are shown in figure 4.

Finally, the Single Top production would inevitably sign New Physics since the production in the Standard Model is smaller than $10^{-9} \mathrm{fb}[8]$. The absence of signal was interpreted in terms of CI by Delphi [9] and L3 [10]. The Non Standard cross-section can include tensor, vectorial and scalar contributions [11]. Limits ranging between 0.5 to $1.3 \mathrm{TeV}$ are obtained depending on the models.

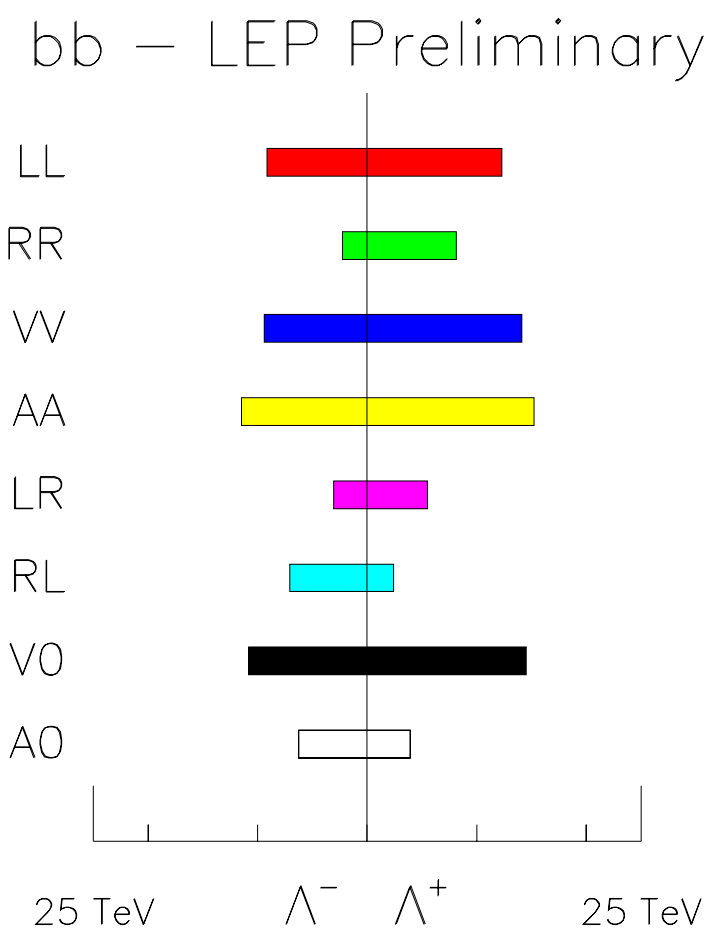

Fig. 3. Limits on the scale of CI in $e^{+} e^{-} \rightarrow b \bar{b}$ using LEP combined measurements from 130 to $209 \mathrm{GeV}$

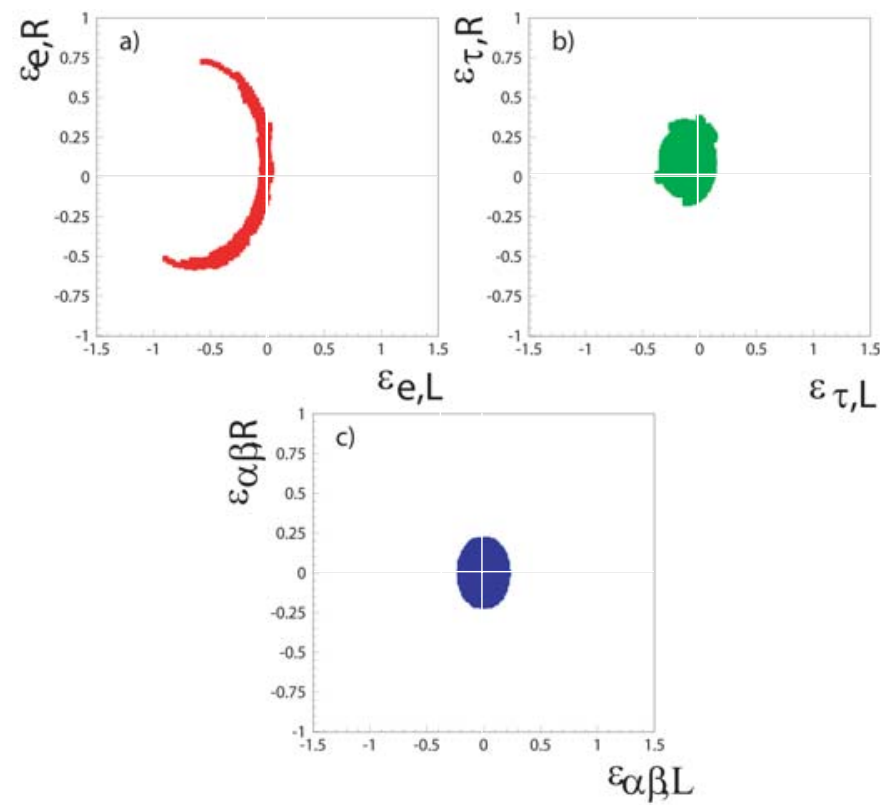

Fig. 4. DELPHI PRELIMINARY: Exclusion contour for CI involving neutrinos final state where $\mathrm{L} / \mathrm{R}$ refers to the helicity of the incoming electrons. a) The region in the $\varepsilon_{e L}-\varepsilon_{e R}$ plane which is not excluded b) The region in the $\varepsilon_{\tau L}-\varepsilon_{\tau R}$ plane which is not excluded. c) The region in the $\varepsilon_{\alpha \beta L}-\varepsilon_{\alpha \beta R}(\alpha \neq \beta)$ plane which is not excluded. 


\section{Interpretations in terms of specific models}

Although Contact interactions were initially formulated in the scope of compositeness, they can be interpreted in other specific extensions of the Standard Model. A selection are reviewed in the following subsections.

\subsection{Extra Z Bosons}

Additional heavy gauge bosons, Z', are predicted by many theories. Indirect effects could be seen at LEP in the combined hadronic and leptonic cross-sections and asymmetries. Table 2 presents lower limits on the Z' mass for some specific models $\chi, \psi, \eta$ from the E6-GUT group, for L$\mathrm{R}$ extension group and for a Sequential Standard Model (SSM) Z'.

Table 2. 95\% confidence level lower limits on the Z' mass and $\mathrm{E}_{6}(\chi), E_{6}(\psi), E_{6}(\eta)$, L-R and SSM models [5]

\begin{tabular}{llllll}
\hline Model: & $\chi$ & $\psi$ & $\eta$ & L-R & SSM \\
\hline $\mathrm{m}_{Z^{\prime}}^{l} i m(\mathrm{GeV})$ & 673 & 481 & 434 & 804 & 1787 \\
\hline
\end{tabular}

\subsection{Extra Dimensions}

Recently, theories of quantum gravity with extra spatial dimensions have suggested a way to go around the hierarchy problem [12]. The graviton would propagate in $4+$ $\mathrm{n}$ compactified spatial dimensions resulting in a KaluzaKlein tower, whereas the SM particles stay in the usual dimensions. This results in an effective Planck scale which could as low as the electroweak scale. At colliders, the KK graviton could be exchanged between fermions pairs. Within the "Hewett" scheme [13], preliminary limits on the cut-off $M_{s}$ using the LEP combined di-electron differential cross-sections [5] is $1.2 \mathrm{TeV}$ (resp. 1. TeV) supposing negative (resp. positive) interference. Final H1 limits [2] in the "GRW" scheme [14] are respectively 0.780 and 0.820 $\mathrm{TeV}$.

\subsection{Leptoquarks}

Leptoquarks would mediate lepton-quark transitions. Withi the effective model [15], 14 types are identified, distinguishing coupling to either L or R leptons exclusively. Following the basis in [16], leptoquarks could be exchanged in the processes $\mathrm{e}^{+} e^{-} \longrightarrow q \bar{q}$ at LEP and $\mathrm{e}^{ \pm}(u / d) \longrightarrow e^{ \pm}(u / d)$ at Hera. Limits on the mass of the leptoquark over its coupling are given in table 3 . It should be noted that LEP and Hera provide complementary limits.
Table 3. $95 \%$ confidence level lower limits on $M_{L Q}(G e V) / g$. A - indicates that no limits could be set. NB: The quoted LEP numbers are extrapolated from limits given for a coupling $\sqrt{4 \pi \alpha}$

\begin{tabular}{lll}
\hline LQ type & H1 & LEP \\
& Final limits $[2]$ & Prelim. [5] \\
\hline$S_{0}(L)$ & 710 & 2150 \\
$S_{0}(R)$ & 640 & 1700 \\
$\tilde{S}_{0}(R)$ & 330 & 660 \\
$S_{1}(L)$ & 490 & 1190 \\
$S_{1 / 2}(L)$ & 850 & 590 \\
$S_{1 / 2}(R)$ & 370 & 770 \\
$\tilde{S}_{1 / 2}(L)$ & 430 & - \\
$V_{0}(L)$ & 730 & 3020 \\
$V_{0}(R)$ & 580 & 540 \\
$\tilde{V}_{0}(R)$ & 990 & 1610 \\
$V_{1}(L)$ & 1360 & 2170 \\
$V_{1 / 2}(L)$ & 420 & 1000 \\
$V_{1 / 2}(R)$ & 950 & 750 \\
$\tilde{V}_{1 / 2}(L)$ & 1020 & 580 \\
\hline
\end{tabular}

\section{Conclusion}

Indirect searches did not reveal New Physics so far. Tevatron with its upgraded energy and Hera with its specific polarisation program will probe the Standard Model further.

\section{References}

1. E. Eichten et al., Phys. Rev. Lett. 50, (1983) 811.

2. The H1 Collaboration, C. Adloff et al., submitted to Phys. Lett. B (hep-ex/0305015).

3. F. Abe et al., The CDF Collaboration, Phys. Rev. Lett. 79, (1997) 2198.

4. B. Abbott et al., The D0 Collaboration, Phys. Rev. Lett. 82, (1999) 2457.

5. The LEP Collaborations, LEP2ff/02-03.

6. The Delphi Collaboration, DELPHI 2003-040/CONF660.

Contributed Paper for EPS HEP 2003, Abs $\sharp 257$

7. Z. Berezhiani and A. Rossi, DFAQ-01/TH/08, (hepph/0111137).

8. C.-S. Huang, X.-H. Wu and S.-H. Zhu, Phys. Lett. B 452, (1999) 143.

9. The Delphi Collaboration, DELPHI 2001-087/CONF 515 Contributed Paper for EPS HEP 2001 and LP01.

10. The L3 Collaboration, CERN-EP/2002-065, submitted to Phys. Lett. B.

11. S. Bar-Shalom and J. Wudka, Phys. Rev. D 60, (1999) 0940016.

12. N. Arkani-Hamed et al., Phys. Lett. B 429, (1998) 263.

13. J. Hewett, Phys. Rev. Lett. 82, (1999) 4765.

14. G.F. Giudice, R. Rattazzi and J.D. Wells, Nucl. Phys. B 544, (1999) 3 (corr. in hep-ph/9811291 v2).

15. W. Buchmüller et al., Phys. Lett. B 191, (1987) 442. Erratum-ibid 448, (1999) 320.

16. J. Kalinowski et al., Z. Phys. C 74, (1997) 595 (hep$\mathrm{ph} / 9703288)$. 